\title{
An Improved Transmission Strategy for Multiple Antenna Channels with Partial Feedback
}

\author{
June Chul Roh and Bhaskar D. Rao \\ Department of Electrical and Computer Engineering \\ University of California, San Diego \\ La Jolla, CA 92093-0407 \\ E-mail: jroh@ece.ucsd.edu, brao@ece.ucsd.edu
}

\begin{abstract}
We investigate transmission strategies for multiple antenna systems with partial spatial information of channel available at the transmitter. A new beamforming method is proposed in which the beamforming matrix is determined from the partial spatial information in a novel but predefined way; as a result, the receiver also knows the beamforming matrix. With this beamforming scheme, we develop a multiple antenna system concept that enables better use of the multiple-input multiple-output (MIMO) channels from a channel capacity perspective. The results show that the proposed methods lead to systems wherein the amount of channel feedback information can be significantly reduced with a minor sacrifice of achievable transmission rate.
\end{abstract}

\section{INTRODUCTION}

In recent years, communication systems with multiple antennas at both the transmitter and the receiver have gathered much attention for high-rate data transmission. The informationtheoretical capacity of the multiple antenna channels has been studied by many researchers, e.g., [1], [2]. Many previous studies have focused on the following two assumptions about channel state information (CSI): the first is the case where CSI is known to both the receiver and the transmitter [1], [3]; and the second is where CSI is available only at the receiver, not at the transmitter [1], [2], [4]. We will refer to the former as complete CSIT and the latter as no CSIT $^{1}$; these will be used as the two references in comparing channel capacities.

We remark that there are gaps between the capacities of the two cases, in particular, when the transmit power is relatively low, or when the number of transmit antennas is greater than the number of receive antennas. In order to achieve the higher capacity of the complete CSIT, the transmitter should perfectly know instantaneous channel information. This research was motivated by a natural insight that there is a trade-off between the improvement in channel capacity and the degree of completeness of the CSI available at the transmitter. In practical situations, particularly, in systems with a feedback channel for the channel state information, the amount of channel information that is required at the transmitter can be too large to handle,

\footnotetext{
This research was supported by CoRe research grant No. CorO0-10074 and by a research grant from Ericsson.

${ }^{1}$ CSIT: channel state information at the transmitter. In this paper, we assume that in all cases the channel information is completely known to the receiver.
}

since the channel has $t \times r$ number of fading parameters. In this study, we consider the cases where the channel information is partially known to the transmitter in a way that enables a reduction in the amount of the feedback information. There were several studies that have considered partial CSIT for different systems or in different forms of CSIT other than what this paper considers, e.g., [5], [6], [7], [8], and [9].

There are many applications in which there exists a feedback channel for the channel state information. However, in many real systems, the channel information can not be fully provided to the transmitter, for example, due to a limited transmission capacity of feedback channel or rapid channel variation. In designing such systems, it is important to determine what type of the channel information to feed back while minimizing the loss of channel capacity. In this paper, we consider flat-fading channels with $t$ transmit and $r$ receive antennas which is modeled by an $r \times t$ complex matrix $H$. We propose a beamforming method in which the beamforming matrix is determined from a subset of the eigenvectors of $H^{\dagger} H$ in some predefined way; as a result, the receiver also knows the beamforming matrix. With this beamforming scheme, we introduce a new multiple antenna system concept that provides a mechanism to reduce the amount of channel feedback information. It is shown that this system concept leads to schemes wherein the amount of feedback information can be significantly reduced with a minor sacrifice of achievable transmission rate.

\section{System MOdEL AND BACKGROUND}

\section{A. Channel Model}

We consider multiple antenna systems with $t$ antennas at the transmitter and $r$ at the receiver. Assuming slow flat-fading, the MIMO channel is modeled by the channel matrix $H \in \mathbb{C}^{r \times t}$. That is, the channel input $x \in \mathbb{C}^{t}$ and the channel output $y \in \mathbb{C}^{r}$ have the following relationship:

$$
y=H x+\eta
$$

where $\eta \in \mathbb{C}^{r}$ is the complex additive white Gaussian noise (AWGN) vector with each element being assumed i.i.d. complex Gaussian random variable with zero-mean and unit variance, i.e., $E\left\{\eta \eta^{\dagger}\right\}=I_{r}$. We denote the rank of $H$ by $m$. 
And the singular value decomposition (SVD) of $H$ is given by $H=U \Sigma V^{\dagger}$, where $A^{\dagger}$ denotes the conjugate transpose of a matrix $A$; unitary matrices $V \in \mathbb{C}^{t \times t}$ and $U \in \mathbb{C}^{r \times r}$ span the input space $\mathbb{C}^{t}$ and the output space $\mathbb{C}^{r}$, respectively; and $\Sigma \in \mathbb{R}^{r \times t}$ contains the singular values with $\sigma_{i}$ representing the $i$-th singular value of $H$ and $\sigma_{1} \geq \ldots \geq \sigma_{m}>0$. We impose a constraint on the transmit power, $E\left\{x^{\dagger} x\right\} \leq P_{T}$.

In this paper, we assume that in all cases perfect CSI is known to the receiver. In addition, it is assumed that the transmitter knows the first $n$ column vectors of $V$, where $0 \leq n \leq m$, or the first $n$ eigenvectors of $H^{\dagger} H$, as partial spatial information of the channel. This assumption includes the two extreme cases: i) $n=m$ is the case that the transmitter has same spatial information as in the complete CSIT case; and ii) $n=0$ accounts that no spatial information is available at the transmitter as in the no CSIT case. This paper mainly considers the cases of $0<n<m$; these corresponds to partial CSIT cases. For notational convenience, let us define $V_{1}=\left[v_{1}, \ldots, v_{n}\right]$ where $v_{i}$ is the $i$-th column vector of $V$, and $V_{2}=\left[v_{n+1}, \ldots, v_{t}\right]$, i.e., $V=\left[V_{1}, V_{2}\right]$.

\section{B. MIMO Channels with Complete and No CSIT}

We consider the ergodic capacity [1] as a performance measure. The ergodic capacity of a random MIMO channel with transmit power constraint $P_{T}$ is given by

$$
C\left(P_{T}\right)=E_{H}\left\{C\left(P_{T} ; H\right)\right\}
$$

where $E_{H}\{\cdot\}$ indicates the expectation over channel realizations; and $C\left(P_{T} ; H\right)$ is the conditional capacity for a given channel realization $H$ with a power constraint $P_{T}$. That is,

$$
C\left(P_{T} ; H\right)=\max _{p(x): E\left\{x^{\dagger} x\right\} \leq P_{T}} I(x ; y) .
$$

The mutual information $I(x ; y)$ satisfies $I(x ; y) \leq \log \operatorname{det}\left(I_{r}+\right.$ $\left.H \Phi_{x} H^{\dagger}\right)$, where $\Phi_{x}=E\left\{x x^{\dagger}\right\}$ and the equality holds if and only if $x$ is a circularly symmetric complex Gaussian random vector.

Let us denote by $C_{H H}$ the capacity of MIMO channels with CSI fully known to both the transmitter and the receiver (complete CSIT); by $C_{\phi H}$ the capacity with CSI known only to the receiver (no CSIT). When the transmitter knows the channel information, the optimum power allocation can be solved by water-filling over $m$ independent spatial channels [1]. The capacity is given by

$$
C_{H H}\left(P_{T}\right)=E_{H}\left\{\sum_{i=1}^{m}\left[\log \left(\nu \lambda_{i}\right)\right]^{+}\right\}
$$

where $\lambda_{i}=\sigma_{i}^{2}$ is the $i$-th largest eigenvalue of $H^{\dagger} H$ (or $H H^{\dagger}$ ), and $\left\{P_{i}\right\}$ are the transmit powers allocated on the transmit symbol $s \in \mathbb{C}^{t}(x=V s)$, i.e., $\Phi_{s}=E\left\{s s^{\dagger}\right\}=\operatorname{diag}\left(P_{1}, \ldots, P_{t}\right)$. $[a]^{+}$is defined as $\max \{a, 0\}$ and $\nu$ is the level of water-filling satisfying the power constraint: $\sum_{i=1}^{m}\left[\nu-\frac{1}{\lambda_{i}}\right]^{+}=P_{T}$.
On the other hand, when the transmitter has no knowledge about the channel, it is optimal to use an equal power allocation [1], i.e., $P_{i}=P_{T} / t$ for $1 \leq i \leq t$. Then, the capacity is given by

$$
C_{\phi H}\left(P_{T}\right)=E_{H}\left\{\sum_{i=1}^{m} \log \left(1+\frac{P_{T}}{t} \lambda_{i}\right)\right\}
$$

\section{New Multiple Antenna System Concept}

\section{A. Extended Maximal-Ratio Transmission}

In a system where single data stream is transmitted over $t$ transmit antennas after passing through a beamformer $w \in \mathbb{C}^{t}$, the optimum choice of $w$ is the first eigenvector of $H^{\dagger} H$, or, $v_{1}$. This transmission scheme is called maximal-ratio transmission (MRT). The choice of the beamformer as $w=v_{1}$ is optimal in terms of maximizing the received signal-to-noise ratio [10]. In addition, it can be easily shown that the beamformer choice of $w=v_{1}$ is also optimal in the sense of maximizing the mutual information.

A generalization of the MRT for a system with $n>1$ data streams is to employ $n$ different beamformers for each data stream. In [11], it was discussed that the optimum beamformer, for the case when $n<m$, is likely to be $V_{1}$. The transmitter uses only the $n$ known spatial channels, that is, transmitting $n$ data streams using the $n$ eigenvectors. We will call this scheme extended MRT. Since the receiver knows the channel parameters, the channel can be decomposed into $n$ parallel channels with different channel gains. As a result, with this strategy, one can employ conventional scalar coding to each spatial channel. But, because the inherent multiplexing capability of the multiple antenna channel is not fully exploited, it will be shown later that this strategy is inferior to the transmission strategies we propose in the following.

\section{B. The Beamforming Method and New Multiple Antenna Sys- tem Concept}

To fully exploit potential multiplexing capability of the channel, we propose a new and improved beamforming method that also utilizes the orthogonal complement of the space spanned by $V_{1}$. A beamforming matrix $W \in \mathbb{C}^{t \times t}$ is generated as a function of $V_{1}$ in a predefined manner. Since the receiver has knowledge of $V_{1}$, the receiver is also aware of the beamforming matrix that the transmitter will use. This property enables us to conceive of a new multiple antenna system concept which is described in the next subsection. One reasonable way to generate the beamforming matrix is the following:

1) Choose $t-n$ vectors, namely, $\tilde{V}_{2}=\left[\tilde{v}_{n+1}, \ldots, \tilde{v}_{t}\right]$, that are mutually orthogonal and also orthogonal to the space spanned by $V_{1}$, i.e.,

$$
\tilde{V}_{2}^{\dagger} \tilde{V}_{2}=I_{t-n}, \quad V_{1}^{\dagger} \tilde{V}_{2}=0
$$

where $I_{p}$ is $p \times p$ identity matrix and 0 is $n \times(t-n)$ zero matrix. 
2) Concatenate $\tilde{V}_{2}$ to $V_{1}$ to form a beamforming matrix $W=\left[V_{1}, \tilde{V}_{2}\right]$.

It can be easily shown that, if $H$ is full rank, $W$ spans the same input space as $V$ does. The beamforming matrix $W$ is used in transmitting the information vector $s \in \mathbb{C}^{t}$ in a manner similar to the use of $V$ in the complete CSIT case. The procedure for selecting $\tilde{V}_{2}$ satisfying (4) can be defined in various ways, e.g., $\tilde{V}_{2}$ are the eigenvectors corresponding to the nonzero eigenvalues of $I_{t}-V_{1} V_{1}^{\dagger}$. Whatever be the mechanism for generating $\tilde{V}_{2}$ at the transmitter, the generating mechanism is assumed to be known at the receiver so that the receiver can also independently generate $\tilde{V}_{2}$ and, hence, $W$.

With the proposed beamforming scheme, we develop a new multiple antenna system concept that can potentially lead to a reduction in the amount of channel feedback information. It involves

1) Based on $W$, calculation for optimal power allocation over transmit symbols is performed at the receiver.

2) The power allocation result is provided to the transmitter as additional CSIT.

The first step above will be described in detail in the next Section. It results in $t$ real values, each is bounded between 0 and 1 , and sum up to be 1 . Then, the total channel feedback information is $n t$-dimensional complex vectors, i.e., $V_{1}$, plus $t$ real values in $[0,1]$. Thus, in most systems, in particular, when the number of transmit antennas $t$ is large, the amount of feedback information can be significantly reduced.

\section{Channel Decomposition}

Now, we will show that by using the proposed beamforming method the original MIMO channel is decomposed into two parts. The transmitted signal $x$ is given by

$$
x=W s=\left[V_{1}, \tilde{V}_{2}\right]\left[\begin{array}{l}
s_{1} \\
s_{2}
\end{array}\right]=V_{1} s_{1}+\tilde{V}_{2} s_{2}
$$

where $s=\left[s_{1}, s_{2}\right]^{T} \in \mathbb{C}^{t}, s_{1} \in \mathbb{C}^{n}$, and $s_{2} \in \mathbb{C}^{(t-n)}$.

The receiver pre-multiplies the received signal $y=H x+\eta$ by $U^{\dagger}$ to have $\tilde{y}=U^{\dagger} y$. Using the partitioned matrices of compatible sizes to $W=\left[V_{1}, \tilde{V}_{2}\right], \tilde{y}$ can be written as follows:

$$
\begin{aligned}
\tilde{y}=\left[\begin{array}{c}
\tilde{y}_{1} \\
\tilde{y}_{2}
\end{array}\right] & =\left[\begin{array}{cc}
\Sigma_{1} & 0 \\
0 & \Sigma_{2}
\end{array}\right]\left[\begin{array}{ll}
V_{1}^{\dagger} V_{1} & V_{1}^{\dagger} \tilde{V}_{2} \\
V_{2}^{\dagger} V_{1} & V_{2}^{\dagger} \tilde{V}_{2}
\end{array}\right]\left[\begin{array}{l}
s_{1} \\
s_{2}
\end{array}\right]+\left[\begin{array}{l}
\tilde{\eta}_{1} \\
\tilde{\eta}_{2}
\end{array}\right] \\
& =\left[\begin{array}{cc}
\Sigma_{1} & 0 \\
0 & \Sigma_{2} V_{2}^{\dagger} \tilde{V}_{2}
\end{array}\right]\left[\begin{array}{l}
s_{1} \\
s_{2}
\end{array}\right]+\left[\begin{array}{l}
\tilde{\eta}_{1} \\
\tilde{\eta}_{2}
\end{array}\right]
\end{aligned}
$$

where $\tilde{y}_{1} \in \mathbb{C}^{n}, \tilde{y}_{2} \in \mathbb{C}^{(r-n)}$, diagonal matrices $\Sigma_{1} \in \mathbb{R}^{n \times n}$ and $\Sigma_{2} \in \mathbb{R}^{(r-n) \times(t-n)}$ contain $\sigma_{1}, \ldots, \sigma_{n}$ and $\sigma_{n+1}, \ldots, \sigma_{m}$, respectively, and zero matrices are of suitable size. Equation (5) results from the facts that $V_{1}^{\dagger} V_{1}=I_{n}, V_{1}^{\dagger} \tilde{V}_{2}=0$, and $V_{2}^{\dagger} V_{1}=$ 0.

We can see that the MIMO channel has been decomposed into $n$ non-interfering parallel channels and a new coupled
MIMO channel with a channel matrix $H_{2}=\Sigma_{2} V_{2}^{\dagger} \tilde{V}_{2}$ in $\mathbb{C}^{(r-n) \times(t-n)}$. That is,

$$
\begin{aligned}
& \tilde{y}_{1}=\Sigma_{1} s_{1}+\tilde{\eta}_{1} \\
& \tilde{y}_{2}=H_{2} s_{2}+\tilde{\eta}_{2}
\end{aligned}
$$

We will refer to the first channel of (6) as the $\Sigma_{1}$ channel, and the second channel of (7) as the $H_{2}$ channel. Note that the covariance of $\tilde{\eta}=U^{\dagger} \eta=\left[\tilde{\eta}_{1}, \tilde{\eta}_{2}\right]^{T}$ is unchanged as $E\left\{\tilde{\eta} \tilde{\eta}^{\dagger}\right\}=I_{r}$. An interesting property about the singular values of the channel matrix is summarized in the following Lemma.

Lemma 1: The singular values of the channel matrix $H_{2}=$ $\Sigma_{2} V_{2}^{\dagger} \tilde{V}_{2}$ is preserved as $\operatorname{diag}\left(\Sigma_{2}\right)$.

Proof: See [12].

By the following Lemma, we show that the mutual information $I(x ; y)$ is preserved with the linear operations $x=\tilde{V} s$ and $\tilde{y}=U^{\dagger} y$. Furthermore, $I(x ; y)$ can be given by the sum of the mutual information expressions for two decomposed channels.

Lemma 2: For a given channel realization $H$, the mutual information between the input and the output of the MIMO channel can be expressed as

$$
\begin{aligned}
I(x ; y) & =I(s ; \tilde{y}) \\
& =I\left(s_{1} ; \tilde{y}_{1}\right)+I\left(s_{2} ; \tilde{y}_{2}\right) .
\end{aligned}
$$

Proof: See [12].

\section{MiMO Channel with Partial CSIT}

In this section, we derive the ergodic channel capacity and the optimum transmit power allocation schemes for the MIMO system with partial CSIT described in the previous sections.

As we discussed in the previous Sections, by using a beamforming matrix generated in a predefined way, the beamforming matrix $W$ is also known to the receiver. In this Section, we discuss the transmission schemes and accompanying optimum power allocation solutions that take advantage of receiver's knowledge of the channel. For simplicity, we define an equivalent channel matrix $A=U^{\dagger} H W$, or $\Sigma V^{\dagger} W$, which represents the channel between $s$ and $\tilde{y}$, i.e., $\tilde{y}=A s+\tilde{\eta}$. By Lemma 2, maximizing $I(x ; y)$ is equivalent to maximizing $I(s ; \tilde{y})$. Thus, since $I(s ; \tilde{y}) \leq \log \operatorname{det}\left(I_{r}+A \Phi_{s} A^{\dagger}\right)$, we have

$$
C_{V_{1} H}^{\text {(opt) }}\left(P_{T} ; H\right)=\max _{\substack{P_{1} \geq 0, \ldots, P_{t} \geq 0 \\ P_{1}+\cdots+P_{t}=P_{T}}} \log \operatorname{det}\left(I_{r}+A \Phi_{s} A^{\dagger}\right)
$$

The above maximization problem is complex, and, except in some special cases, it requires the use of numerical optimization methods. Fortunately, this optimization problem is matched to the so-called determinant maximization problem [13] and can be solved numerically by using MAXDET algorithm [14]. The optimal power allocation for the $\mathrm{H}_{2}$ channel, in general cases, turned out to be unequal even for the symbols over the $H_{2}$ channel. In this scenario, the channel feedback information is $V_{1}$ and the power allocation $\left(\gamma_{1}, \ldots, \gamma_{t}\right)$, where $\gamma_{i}$ is defined as $P_{i} / P_{T}$. We can find closed-form expressions in the following practically important cases. 


\section{A. Case I: when $r=1$ and $n=0$}

In this case, since no eigenvector of $H^{\dagger} H$ is provided to the transmitter, we consider a natural choice of the beamforming matrix, $W=I_{t}$. Then, since the equivalent channel is now a $t$-dimensional row vector denoted by $A=\left[a_{1}, \ldots, a_{t}\right]$, the maximization problem of equation (10) becomes a simple form of the following:

$$
C_{\phi H}^{\text {(opt) }}\left(P_{T} ; H\right)=\max _{\substack{P_{1} \geq 0, \ldots, P_{t} \geq 0 \\ P_{1}+\cdots+P_{t}=P_{T}}} \log \left(1+\sum_{i=1}^{t}\left|a_{i}\right|^{2} P_{i}\right)
$$

By the monotonicity of log function and using a well-known solution in conventional linear programming, we have the following solution:

$$
C_{\phi H}^{(\mathrm{opt})}\left(P_{T} ; H\right)=\log \left(1+\left|a_{i^{*}}\right|^{2} P_{T}\right)
$$

where $i^{*}=\arg \max _{i}\left|a_{i}\right|^{2}$. This means that the total transmit power should be allocated such that $P_{i^{*}}=P_{T}$ and $P_{i}=0$ for all $i \neq i^{*}$.

The above analysis indicates that if the index for $i^{*}$ is available at the transmitter via some feedback we can obtain higher channel capacity than that of no CSIT case. This can be interpreted as transmit antenna selection method in which at a given time only one antenna that provides the best link to the receiver is used in transmitting data.

\section{B. Case II: when $t \geq r$ and $n=r-1$}

An example of this case is $r=2, n=1$, and $t \geq 2$. In this case, the equivalent channel $A$ is given by

$A=\left[\begin{array}{cccccccc}\sigma_{1} & 0 & \cdots & 0 & 0 & 0 & \cdots & 0 \\ 0 & \sigma_{2} & 0 & \vdots & \vdots & \vdots & \vdots & 0 \\ 0 & 0 & \ddots & 0 & 0 & 0 & \cdots & 0 \\ 0 & \cdots & 0 & \sigma_{r-1} & 0 & 0 & \cdots & 0 \\ 0 & \cdots & 0 & 0 & \sigma_{r} \alpha_{r, r} & \sigma_{r} \alpha_{r, r+1} & \cdots & \sigma_{r} \alpha_{r, t}\end{array}\right]$

where $\alpha_{i, j}$ is defined as the inner product between the $i$-th column of $V$ and the $j$-th column of $W$, i.e., $\alpha_{r, j}=v_{r}^{\dagger} \tilde{v}_{j}$ for $r \leq j \leq t$. Therefore, the channel can be expresses as follows:

$\tilde{y}_{i}=\left\{\begin{array}{l}\sigma_{i} s_{i}+\tilde{\eta}_{i}, \quad \text { for } i=1, \ldots, r-1 ; \\ \sigma_{r}\left(\alpha_{r, r} s_{r}+\alpha_{r, r+1} s_{r+1}+\ldots+\alpha_{r, t} s_{t}\right)+\tilde{\eta}_{r}, i=r\end{array}\right.$

where $\tilde{y}_{i}$ denotes the $i$-th component of the $r$-dimensional vector $\tilde{y}$ and $s_{i}$ the $i$-th symbol of $s .^{2}$

Then, applying the same linear optimization technique as in the previous subsection to the last MISO channel (the input are $s_{r}, \ldots, s_{t}$ and the output is $\tilde{y}_{r}$ ), the maximization problem of

\footnotetext{
${ }^{2}$ Note the definitions of $\tilde{y}_{i}$ and $s_{i}$ are different from that in Section III.
}

(10) becomes the following:

$$
\begin{aligned}
& C_{V_{1} H}^{\text {(opt) }}\left(P_{T} ; H\right)= \max _{\substack{P_{1} \geq 0, \ldots, P_{r-1} \geq 0, P_{j^{*}} \geq 0 \\
P_{1}+\cdots+P_{r-1}+P_{j^{*}}=P_{T}}} \\
&\left\{\sum_{i=1}^{r-1} \log \left(1+\sigma_{i}^{2} P_{i}\right)+\log \left(1+\sigma_{r}^{2}\left|\alpha_{r, j^{*}}\right|^{2} P_{j^{*}}\right)\right\}
\end{aligned}
$$

where $j^{*}=\arg \max _{r \leq j \leq t}\left|\alpha_{r, j}\right|^{2}$. Notice that the channel we consider consists of $r$ parallel Gaussian channels with each channel having a channel gain, $\sigma_{1}, \ldots, \sigma_{r-1}, \sigma_{r}\left|\alpha_{r, j^{*}}\right|$, respectively. Thus, the conditional channel capacity can be solved by conventional water-filling over the $r$ noisy channels with equivalent noise levels of $\left(1 / \sigma_{1}^{2}, \ldots, 1 / \sigma_{r-1}^{2}, 1 / \sigma_{r}^{2}\left|\alpha_{r, j^{*}}\right|^{2}\right)$.

The above result implies that the channel feedback information is $V_{1}$ and the index for $j^{*}$ that indicates the antenna, among the antenna set of $r$-th to $t$-th antenna, that provides the best link to last channel output $\tilde{y}_{r}$.

\section{NUMERICAL RESUlts}

For comparative studies, we consider the extended MRT scheme. The transmitter is also assumed to know $V_{1}$, and only $n$ spatial channels are used for transmission. That is, all the transmit power $P_{T}$ is allocated only to the $\Sigma_{1}$ channel in a water-filling manner. It is equivalent to the case where the $H_{2}$ channel in our system model is not used. Since the extended MRT scheme abandons a chance for the additional potential gain from the $H_{2}$ channel, it is surely inferior to the proposed scheme. By looking at the problems in points of optimization, we can easily see the following relationships between capacities with different CSI assumptions and transmission strategies.

$$
\begin{gathered}
C_{\phi H}\left(P_{T}\right) \leq C_{V_{1} H}^{(\text {opt }}\left(P_{T}\right) \leq C_{H H}\left(P_{T}\right) \\
C_{V_{1} H}^{\text {(EMRT) }}\left(P_{T}\right) \leq C_{V_{1} H}^{\text {(opt) }}\left(P_{T}\right) \leq C_{H H}\left(P_{T}\right)
\end{gathered}
$$

Although the proposed system is irrelevant to the channel model, for numerical comparisons, we considered the MIMO channel that was assumed in [1]. The channel gain matrix $H \in \mathbb{C}^{r \times t}$ is a random matrix independent to the transmit symbols $s$ and the additive noise $\eta$, with i.i.d. entries, each having independent real and imaginary parts with zero-mean and variance $1 / 2$.

Figure 1 and 2 show ergodic capacities for the optimum transmission scheme, with parameters $(t=4, r=2)$ and $(t=$ $6, r=3)$, respectively. The conditional capacity $C_{V_{1} H}^{(\mathrm{opt})}\left(P_{T} ; H\right)$ was calculated using the MAXDET (determinant maximization) algorithm [13]. The following asymptotical behaviors of $C_{V_{1} H}$ can be seen.

$$
C_{V_{1} H}^{(\mathrm{opt})}\left(P_{T}\right) \rightarrow C_{H H}\left(P_{T}\right), \text { as } P_{T} \rightarrow 0
$$

This is because when the transmit power is low, by water-filling, in most case the power is allocated only to first a few channels and nothing to the $\Sigma_{2}$ channel; therefore, the two capacities 
are similar. This shows that spatial information is more important in low range of transmit power. By comparing $C_{V_{1} H}^{\text {(opt) }}$ and $C_{V_{1} H}^{(\mathrm{EMRT})}$, it is observed that at low transmit power the two are very close, and as the transmit power increases $C_{V_{1} H}^{(\mathrm{opt})}$ is getting larger than $C_{V_{1} H}^{(\mathrm{EMRT})}$. From this observation, we can say that the $\mathrm{H}_{2}$ channel should be utilized to achieve higher capacity at medium and high range of transmit power.

\section{CONCLUSION}

We considered multiple antenna systems consisting of $t$ transmit and $r$ receive antennas, and partial channel state information available to the transmitter. When the multiple antenna channel is represented by a channel matrix $H$ in $\mathbb{C}^{r \times t}$, the first $n$ eigenvectors of $H^{\dagger} H$ are assumed to be available at the transmitter as partial spatial information of the channel. We proposed a novel transmission strategy in which a beamforming matrix is determined from the $n$ eigenvectors in a predefined way. From the beamforming method, a new multiple antenna system concept was developed which enables better use of the MIMO channels. The ergodic channel capacity and accompanying power allocation solution have been derived. The simulation results have shown that, in moderate transmit power region, the proposed multiple antenna systems with partial channel information give channel capacities close to those with full channel information at the transmitter. In practical applications that need a feedback for the channel information, the amount of feedback information can be significantly reduced with a minor sacrifice of channel capacity by using the proposed schemes.

\section{REFERENCES}

[1] I. E. Telatar, "Capacity of multi-antenna Gaussian channels," AT\&T Bell Labs Tech. Memo., 1995.

[2] G. J. Foschini and M. J. Gans, "On limits of wireless communications in a fading environment when using multiple antennas," Wireless Personal Communications, vol. 6, pp. 311-335, Mar. 1998.

[3] E. Biglieri, G. Caire, and G. Taricco, "Limiting performance of blockfading channels with multiple antennas," IEEE Trans. Inform. Theory, vol. 47, pp. 1273-1289, May 2001.

[4] A. Narula, M. D. Trott, and G. W. Wornell, "Performance limits of coded diversity methods for transmitter antenna arrays," IEEE Trans. Inform. Theory, vol. 45, pp. 2418 - 2433, Mar. 1999.

[5] G. Caire and S. Shamai, "On the capacity of some channels with channel state information," IEEE Trans. Inform. Theory, vol. 45, pp. 2007-2019, Sept. 1999

[6] E. Visotsky and U. Madhow, "Space-time transmit precoding with imperfect feedback," IEEE Trans. Inform. Theory, vol. 47, pp. 2632-2639, Sept. 2001.

[7] S. A. Jafar, S. Vishwanath, and A. Goldsmith, "Channel capacity and beamforming for multiple transmit and receive antennas with covariance feedback," in Proc. IEEE ICC 2001, pp. 2266 -2270.

[8] S. Bhashyam, A. Sabharwal, and B. Aazhang, "Feedback gain in multiple antenna systems," IEEE Trans. on Commun., vol. 50, pp. 785-798, May 2002.

[9] G. Jongren, M. Skoglund, and B. Ottersten, "Combining beamforming and orthogonal space-time block coding," IEEE Trans. Inform. Theory, vol. 48, pp. 611-627, Mar. 2002.

[10] T. K. Y. Lo, "Maximum ratio transmission," IEEE Trans. Commun., vol. 47 , pp. $1458-1461$, Oct. 1999.
[11] H. Sampath, P. Stoica, and A. Paulraj, "Generalized linear precoder and decoder design for MIMO channels using the weighted MMSE criterion," IEEE Trans. Commun., vol. 49, pp. 2198-2206, Dec. 2001.

[12] J. C. Roh and B. D. Rao, "Multiple antenna channels with partial channel state information at the tarnsmitter," submitted to IEEE Trans. Wireless Commun., Oct. 2002.

[13] L. Vandenberghe, S. Boyd, and S.-P. Wu, "Determinant maximization with linear matrix inequality constraints," SIAM J. Matrix Anal. Applic., vol. 19, pp. 499-533, Apr. 1998.

[14] S.-P. Wu, L. Vandenberghe, and S. Boyd, "MAXDET: Software for determinant maximization problems: User's guide, alpha version." Stanford Univ., Apr. 1996.

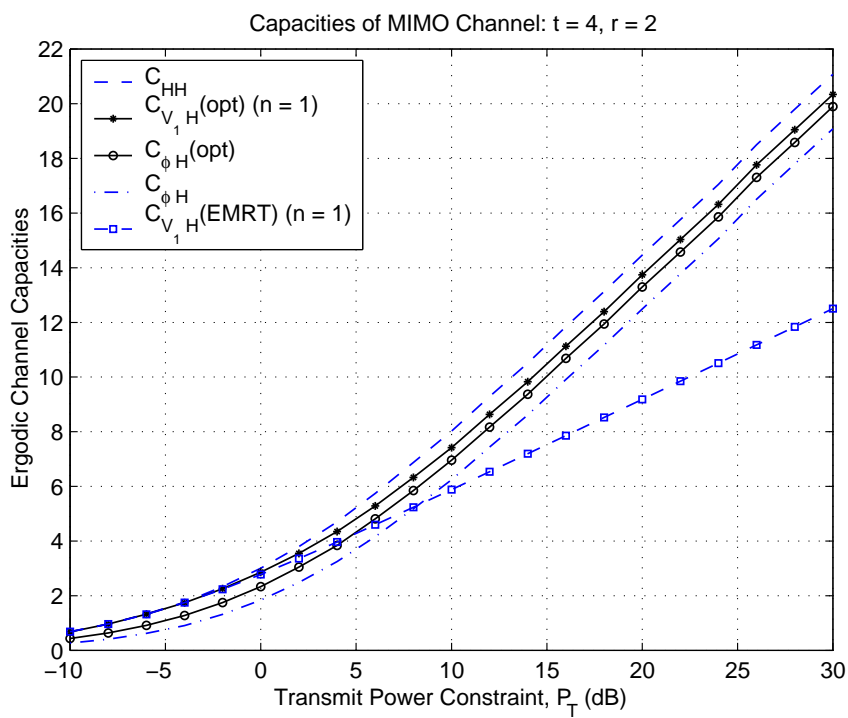

Fig. 1. Ergodic capacities of MIMO channel with different CSI assumptions and transmission strategies ( $t=4$ and $r=2$ ).

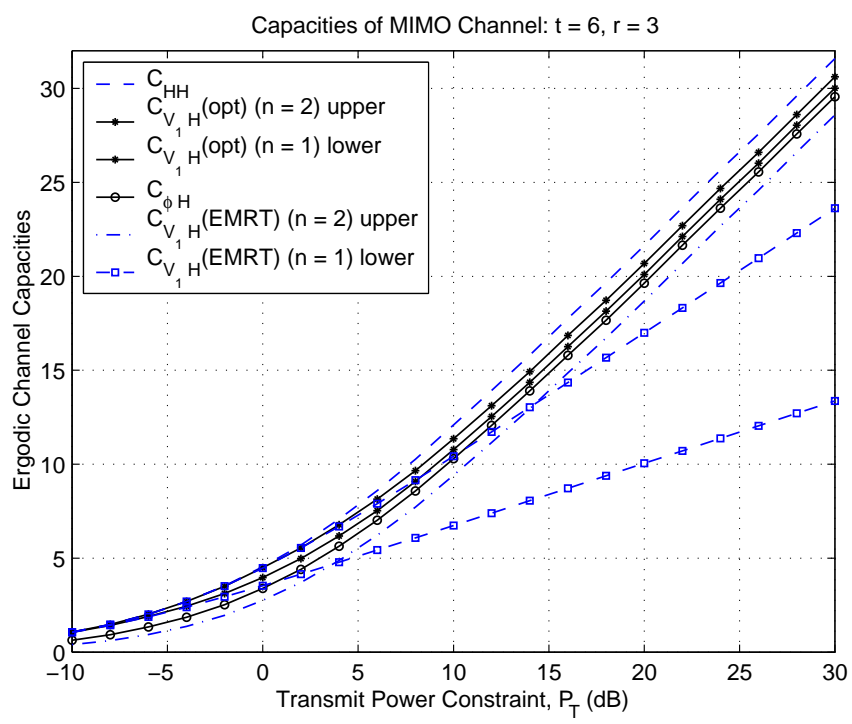

Fig. 2. Ergodic capacities of MIMO channel with different CSI assumptions and transmission strategies ( $t=6$ and $r=3$ ). 